\title{
EFFECT OF LEAD DISTANCE ON THE TRANSMITTANCE OF THE LIGHT THROUGH MORTAR MADE OUT OF OPTIC FIBERS
}

\author{
Joice John ${ }^{1}$, S. J Krishna Prasad ${ }^{2}$, R.Prabhakara ${ }^{3}$ \\ ${ }^{1}$ M.Tech Student in Civil Engg Department MSRIT, Bangalore \\ ${ }^{2}$ Associate. Professor of the Department of Telecommunication, MSRIT, Bangalore \\ ${ }^{3}$ Professor and Head of the Department of Civil Engineering, MSRIT, Bangalore
}

\begin{abstract}
The aim of this project was to produce functional concrete by incorporating optical fibers, which are known to have an excellent light transmitting property. The final product is known as translucent concrete. This is used in various architectural, interior design applications such as partition walls, wall claddings and dividers. It has been observed from the literature that translucent mortar using $2.36 \mathrm{~mm}$ downsize fine aggregate with the optical fibers are becoming very popular due to mould-ability with good quality control without damaging the optical fibers. In the present investigation, the transmittance of light was studied to observe the variation with respect to the low, medium and high intensity light passing through it. The lead distance is also an important parameter, which has been evaluated by keeping the source of the light at a distance of 70cm, to describe the variation in the transmittance. Experimental investigation has been carried out by casting 3 mortar specimens of MasterEmaco $S 348$ with $0.75 \mathrm{~mm}$ dia optical fibers in $4 \%$ by volume, to observe the above parameters.
\end{abstract}

Keywords- Energy Savings, Translucent Concrete, Transmittance

\section{INTRODUCTION}

In the recent past due to the fast development, the usage of non renewable source of energy has increased. Emphasis is being given on usage of smart materials because; the smart materials have the property to react to environmental changes such as temperature, light, pressure or electricity. Thus, the smart materials can be used in the green buildings. Countries like China and Japan are focusing on variation of electrical energy for power lighting. Electricity being one of the most important forms of energy, the depletion of coal, which is used for generation of electricity, makes it very necessary to save electricity.

In 2001, Aaron Losonczi, a Hungarian [1] architect, used glass fibers in concrete to transmit light naturally or artificially. Thus concrete, which is the most widely used material for the development of infrastructure and housing is combined with optical fiber, which has excellent light guiding properties in the production of the translucent concrete. The main purpose of transparent block is to use sunlight as the source of light instead of using electrical energy. In other words variation of operating energy by exploiting vast amount of potential energy in the form of sunlight, which can result in energy saving.

This current study was an inter departmental project. Mechanical department of MSRIT helped in the manufacture of the moulds with perforations. The cubes were casted and tested in the Civil Engineering department. Telecommunication department provided the guidance and instruments for the transmittance test.

\section{LITERATURE REVIEW}

Akshaya B Kamdi [2] a research paper documented on the manufacturing, uses and future scope of translucent concrete. Aggregates were replaced with optical fibers. Optical fibers blended into the concrete like small pieces of aggregate; the concrete with optical fiber had same strength as that of a normal concrete cube and it was frost, fire and UV resistant. 2 micron to $2 \mathrm{~mm}$ diameter optical fiber was used in 4 to $5 \%$ by volume in the concrete mix. The placing of optical fiber was time consuming and costly. The mixture was made with fine aggregates only.

They had discussed the use of translucent concrete in various fields. It was concluded that homes could use less light during the day time and hence save energy.

Ravivarman, Mageswari and Kanagalakshmi[3] believed that deriving natural light in buildings was a problem, it gave rise to artificial consumption of energy, which made it very essential to reduce the artificial light consumption in structure. $150 \mathrm{~mm} * 150 \mathrm{mmm} * 150 \mathrm{~mm}$ sized cubes with 4$5 \%$ optical fibers were made and compression test was undertaken. 28 days compression test was noted to be $25 \mathrm{MPa}$, which meant that the concrete was structurally efficient.

Translucent concrete blocks were highly advantageous. Large and tall office buildings could share the lighting if the ceilings were translucent. It was a smart way of optimizing and utilizing light. It was observed that the translucent concrete could be used as panels in slabs, walls. If the 
translucent concrete was used in place of windows, it could transmit the sunlight. Hence, the application of optical fiber would make the concrete decorative as well as energy saving.

A.A. Momin, R.B. Kadiranaikar, Vakeel. S. Jagirdar and Arshad Ahemed Inamdar [4] in this paper they have produced concrete with glass rods and optical fiber as reinforcement with different percentages. The concrete mixture was made up of fine materials. Light transmittance test and compression test were undertaken.

It was observed that transmittance of light was more with less spacing in glass rod and optical fiber but optical fiber had more transmittance compared to glass rod , 7-10\% for optical fiber and $0.2-1.5 \%$ for glass rod .

With the increase in spacing, the transmittance of light in glass fiber decreased by $83.8 \%$ and in case of optical fiber it decreased by $22 \%$.

Karandikar, Virdhi and Deep [1] discussed about translucent concrete and results of the transmittance test done. The main aim of their paper was to design translucent concrete blocks with optical fibers. The optical fibers in $0 \%$, $0.09 \%, 0.87 \%, 1.05 \%$ and $1.75 \%$ of volume were used to analyze the transmittance. The manufacturing process of translucent concrete was almost the same as that of normal concrete. Transmittance test was performed on the cubes. Cubes with $0 \%$ and $0.09 \%$ optical fiber had zero transmittance and cubes with $0.87 \%$ OF had $0.139,1.05 \%$ OF had 0.34 and $1.75 \%$ had 1.27 transmittance.

It was concluded that the as the percentage of optical fiber increased the transmittance increased. The increase in transmittance was noted to be $59 \%$.

Findings:-From the available literature reviews, it was concluded that, when optical fiber was used in the concrete the concrete gave the desirable compressive strength and the cube transmitted light. Hence, production of functional concrete is possible. This could be used as a smart material. The literatures were is available on the transmittance test when the source of light was a constant. In this paper, we are going to discuss the effect of intensity of light by varying the lead distance and by varying the intensity of light.

\section{MATERIAL TESTING}

Optical fibers were bought and they came with the datasheet and the properties are tabulated in table 1 . The core of the fiber used was made up of polymethyl and methacrylate, the cladding is made of fluroresin. It had a multi mode step index, the refractive index of the core was 1.49 and the refractive index of the cladding was 1.41.The Table 1 given below lists the performance based properties of the optical fiber.
Table 1: Properties of $0.75 \mathrm{~mm}$ dia Optical Fiber

\begin{tabular}{|l|l|l|}
\hline $\begin{array}{l}\text { Optical } \\
\text { Performance }\end{array}$ & $\begin{array}{l}\text { Attenuation At 650 } \\
\mathrm{nm}\end{array}$ & $\leq 0.3 \mathrm{Db} / \mathrm{m}$ \\
\hline $\begin{array}{l}\text { Mechanical } \\
\text { Performance }\end{array}$ & Tensile Strength & $\geq 32 \mathrm{~N} / \mathrm{mm}^{2}$ \\
\cline { 2 - 3 } & Yield elongation & $\geq 4 \%$ \\
\cline { 2 - 3 } & $\begin{array}{l}\text { Minimum Bending } \\
\text { Radius }\end{array}$ & $\geq 7.5 \mathrm{~mm}$ \\
\hline
\end{tabular}

To achieve flowable mortar MasterEmaco S 348 was used. This was a product from BASF, which is a chemical based company. It appeared to be grey, it was mainly made up of cement, sand, fibers and acrylic based products to compensate shrinkage. It was thick when water was mixed, but when shook it could flow through tiny gaps. The aggregates present were $2.36 \mathrm{~mm}$ downsize, 0.16 was the water to powder ratio used.The specific gravity of the powder was 2.273.The control specimen had a compressive strength of $63 \mathrm{MPa}$. On addition of fiber in $4 \%$ by volume, the compressive strength was noted to be $51.08 \mathrm{MPa}$

\section{PREPARATION OF SPECIMEN}

The perforations made on the plate were parallel to each other so that the optical fibers could run through those holes from one end to another. In fig 1 shown below, the perforations are clearly visible and the optical fibers, were placed in loops so that it was convenient to hold them in position while pouring the cement mortar in the mould [3]

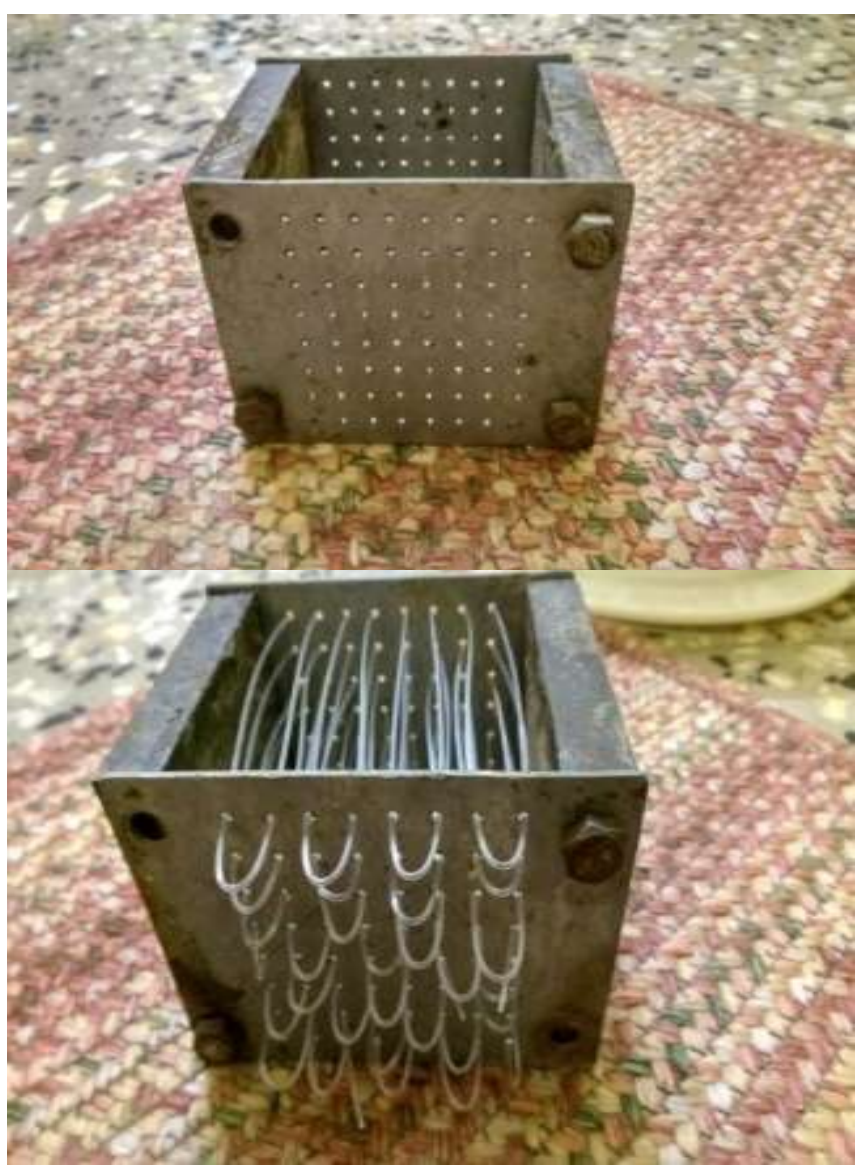

Fig 1: Mould with Perforated Plates on Two Sides 
After placing the optical fiber in the mould as shown in the Figure 1, the mortar was poured in the mould. It was poured in three layers and mechanical vibration was given as it was impossible to tamp due to the presence of optical fibers. The cubes were let to set and demoulding was done after 24 hours. The cubes were kept in the water tub for curing. Grinding machine was used to grind the surface of the cubes to achieve smooth surface for proper transmittance of the light. $0.75 \mathrm{~mm}$ diameter of optical fibers was used to cast three specimens.

\section{EXPERIMENTAL SETUP}

Transmittance test was mainly conducted to find out how much light was being transmitted when passed through the block of translucent concrete. Higher the amount of light passed through the block higher was the transmittance [4]. For the transmittance test, a source of voltage, multi-meter, spring board, resistance and light dependent resistors (LDR), and incandescent bulbs were required. Two readings were taken one without sample (A1) and one with the sample (A2). A resistance of $1 \mathrm{~K}$ was applied in the circuit and a uniform DC voltage of $2.5 \mathrm{v}$ was kept between the circuits. Calculation was done by using the following formula

\section{Light transmittance $=100-[(\mathrm{A} 1-\mathrm{A} 2) / \mathrm{A} 1]^{* 100}$}

Variation in intensity was achieved by using three incandescent bulbs, which were 60w, 100w and 200w, and by varying the lead distance at which the bulbs were placed. The results were tabulated for each of the specimen with variation and the results were tabulated for the entire specimen. The Figure 2 below shows the transmittance test set up.
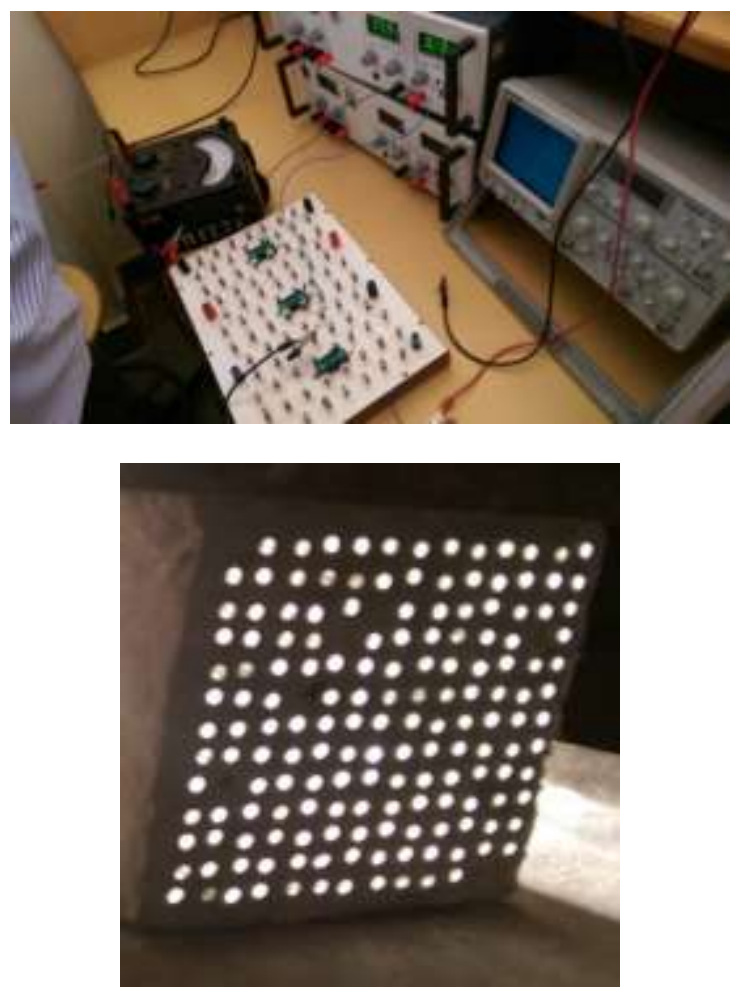

Fig 2:- Transmittance Test Set Up

\section{RESULTS}

\subsection{Transmittance at Different Lead Distance}

The transmittance test was conducted and the results are tabulated in Table 2, when $0.75 \mathrm{~mm}$ dia fibers were used in $4 \%$ by volume. 60 watt, 100 watt and 200 watt bulbs were used for the test.

Table 2:- Variation in Transmittance value at Lead Distance when $60 \mathrm{w}, 100 \mathrm{w}$ and $200 \mathrm{w}$ bulbs were used

\begin{tabular}{|l|l|l|l|}
\hline \multirow{2}{*}{$\begin{array}{l}\text { Lead } \\
\text { Distance }\end{array}$} & \multicolumn{3}{|l|}{ Transmittance Value (\%) } \\
\cline { 2 - 4 } & $\mathbf{6 0 w}$ & $\mathbf{1 0 0 w}$ & $\mathbf{2 0 0 w}$ \\
\hline $\mathbf{1 0 c m}$ & 70.59 & 76.47 & 77.78 \\
\hline $\mathbf{2 0 c m}$ & 68.75 & 60.6 & 76.47 \\
\hline $\mathbf{3 0 c m}$ & 60 & 63.33 & 65.63 \\
\hline $\mathbf{4 0 c m}$ & 42.86 & 55.55 & 59.38 \\
\hline $\mathbf{5 0 c m}$ & 38.46 & 46.15 & 53.33 \\
\hline $\mathbf{6 0 c m}$ & 29.16 & 36.36 & 33.33 \\
\hline $\mathbf{7 0 c m}$ & 20 & 27.27 & 28.57 \\
\hline
\end{tabular}

To show the variation of transmittance with respect to the lead distance, graph has been plotted as shown in Figure 3.

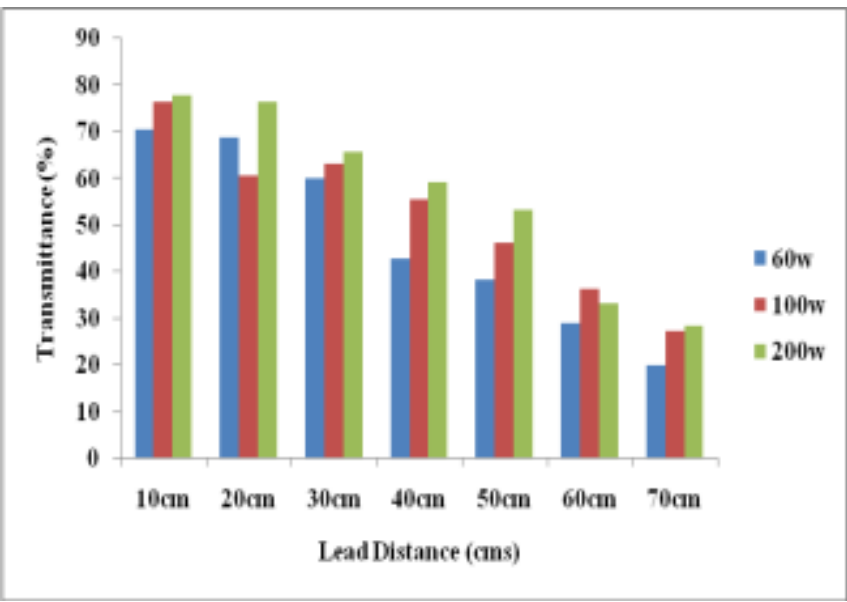

Fig 3:- Variation in Transmittance value at Lead Distance when 60w, 100w and 200w bulb was used

\subsection{Transmittance when Low, Medium and High}

\section{Intensity of Light was Applied}

The different intensity of light was achieved by using bulbs of different power supply. The effect of power supply is tabulated in Table 3.

Table 3:- Variation in Transmittance with respect to the Intensity of light from a distance of $40 \mathrm{~cm}$

\begin{tabular}{|l|l|l|l|}
\hline & $\begin{array}{l}\text { Transmittance } \\
\text { Value }(\mathbf{\%})(\mathbf{6 0 w})\end{array}$ & $\begin{array}{l}\text { Transmittance } \\
\text { Value }(\boldsymbol{\%}) \\
(\mathbf{1 0 0 w})\end{array}$ & $\begin{array}{l}\text { Transmittance } \\
\text { Value }(\mathbf{\%})(\mathbf{2 0 0 w})\end{array}$ \\
\hline $\begin{array}{l}\mathbf{0 . 7 5 m} \\
\mathbf{m}-\mathbf{4 \%}\end{array}$ & 42.86 & 55.55 & 59.38 \\
\hline
\end{tabular}

To demonstrate the variation of transmittance with respect to the intensity if light from a distance of $40 \mathrm{~cm}$, graph has been plotted as shown in figure 4. 
Transmittance Value (60w) Transmittance Value (100w)

Transmittance Value (200w)

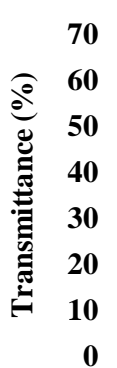

[4]. A.A. Momin, Dr. R.B. Kadiranaikar, Mr.Vakeel.S. Jagirdar, Mr. Arshad Ahemed Inamdar 2014 "Study on Light Transmittance of Concrete Using Optical Fibers and Glass Rods", IOSR Journal of Mechanical and Civil Engineering, pp 67-72

[5]. Jadhav Sunil, Kadlag Amol, Kawade Chetan, Talekar Pravin (2015) "A Study on Translucent Concrete Product and Its Properties by Using Optical Fibers", International open access Journal of Modern Engineering Research, Vol. 5 Iss.4.pp 53-57.

\section{$0.75 \mathrm{~mm}-4 \%$}

\section{Percentage Of Fiber Used By Volume}

Fig 4:- Variation in Transmittance with respect to the Intensity of light from a distance of $40 \mathrm{~cm}$

\section{CONCLUSION}

\subsection{Transmittance at Different Lead Distance}

1. The transmittance was maximal when a 200 watt bulb was used.

2. A reduction in transmittance was being experienced as the lead distance increased.

3. Maximum transmittance was practically possible when $0.75 \mathrm{~mm}$ dia fibers were used in $4 \%$ by volume in the cube.

\subsection{Transmittance when Low, Medium and High}

\section{Intensity of Light was Applied}

1. It can be concluded that high intensity of light (200w) produced maximum transmittance.

2. As the percentage of optical fiber present in the cube increased, the transmittance also increased.

3. It could be concluded that, higher the number of fibers present higher would be the transmittance. $0.75 \mathrm{~mm}$ dia optical fiber used in $4 \%$ by volume had maximum transmittance.

\section{ACKNOWLEDGEMENT}

The fund for the project was provided by Bhagirath Construction Company Address: M.S.R Nagar, Bangalore, Karnataka 560054

\section{REFERENCES}

[1]. Karandikar, N. Virdhi Translucent Concrete (2015) "Test of Compressive Strength and Transmittance", International Journal of Engineering Research \& Technology (IJERT), Vol. 4 Issue 07. Pp 177-180

[2]. Akshaya B Kamdi (August 2013) "Transparent Concrete as a Green Material for Building', Int. J. Struct. \& Civil Engg, Vol. 2, No. 3, pp 172-175

[3]. Ravivarman,Mageswari, and Kanagalakshmi Nov. Dec. 2015),"Experimental study of LiTraCon" IOSR Journal of Mechanical and Civil Engineering, Volume 12, Issue 6 Ver. I, PP 07-13 